\title{
Video Article \\ Evaluation of Biomaterials for Bladder Augmentation using Cystometric Analyses in Various Rodent Models
}

\author{
Duong D. Tu ${ }^{* 1}$, Abhishek Seth ${ }^{{ }^{1}}$, Eun Seok Gil ${ }^{2}$, David L. Kaplan ${ }^{2}$, Joshua R. Mauney ${ }^{1}$, Carlos R. Estrada Jr. ${ }^{1}$ \\ ${ }^{1}$ Children's Hospital Boston, Harvard Medical School \\ ${ }^{2}$ Tufts University \\ *These authors contributed equally
}

Correspondence to: Joshua R. Mauney at joshua.mauney@childrens.harvard.edu, Carlos R. Estrada Jr. at carlos.estrada@childrens.harvard.edu

URL: https://www.jove.com/video/3981

DOI: doi:10.3791/3981

Keywords: Bioengineering, Issue 66, Medicine, Biomedical Engineering, Physiology, Silk, bladder tissue engineering, biomaterial, scaffold, matrix, augmentation, cystometry

Date Published: 8/9/2012

Citation: Tu, D.D., Seth, A., Gil, E.S., Kaplan, D.L., Mauney, J.R., Estrada Jr., C.R. Evaluation of Biomaterials for Bladder Augmentation using Cystometric Analyses in Various Rodent Models. J. Vis. Exp. (66), e3981, doi:10.3791/3981 (2012).

\section{Abstract}

Renal function and continence of urine are critically dependent on the proper function of the urinary bladder, which stores urine at low pressure and expels it with a precisely orchestrated contraction. A number of congenital and acquired urological anomalies including posterior urethral valves, benign prostatic hyperplasia, and neurogenic bladder secondary to spina bifida/spinal cord injury can result in pathologic tissue remodeling leading to impaired compliance and reduced capacity ${ }^{1}$. Functional or anatomical obstruction of the urinary tract is frequently associated with these conditions, and can lead to urinary incontinence and kidney damage from increased storage and voiding pressures ${ }^{2}$. Surgical implantation of gastrointestinal segments to expand organ capacity and reduce intravesical pressures represents the primary surgical treatment option for these disorders when medical management fails ${ }^{3}$. However, this approach is hampered by the limitation of available donor tissue, and is associated with significant complications including chronic urinary tract infection, metabolic perturbation, urinary stone formation, and secondary malignancy ${ }^{4,5}$.

Current research in bladder tissue engineering is heavily focused on identifying biomaterial configurations which can support regeneration of tissues at defect sites. Conventional 3-D scaffolds derived from natural and synthetic polymers such as small intestinal submucosa and polyglycolic acid have shown some short-term success in supporting urothelial and smooth muscle regeneration as well as facilitating increased organ storage capacity in both animal models and in the clinic ${ }^{6,7}$. However, deficiencies in scaffold mechanical integrity and biocompatibility often result in deleterious fibrosis ${ }^{8}$, graft contracture ${ }^{9}$, and calcification ${ }^{10}$, thus increasing the risk of implant failure and need for secondary surgical procedures. In addition, restoration of normal voiding characteristics utilizing standard biomaterial constructs for augmentation cystoplasty has yet to be achieved, and therefore research and development of novel matrices which can fulfill this role is needed.

In order to successfully develop and evaluate optimal biomaterials for clinical bladder augmentation, efficacy research must first be performed in standardized animal models using detailed surgical methods and functional outcome assessments. We have previously reported the use of a bladder augmentation model in mice to determine the potential of silk fibroin-based scaffolds to mediate tissue regeneration and functional voiding characteristics. ${ }^{11,12}$ Cystometric analyses of this model have shown that variations in structural and mechanical implant properties can influence the resulting urodynamic features of the tissue engineered bladders ${ }^{11,12}$. Positive correlations between the degree of matrixmediated tissue regeneration determined histologically and functional compliance and capacity evaluated by cystometry were demonstrated in this model ${ }^{11,12}$. These results therefore suggest that functional evaluations of biomaterial configurations in rodent bladder augmentation systems may be a useful format for assessing scaffold properties and establishing in vivo feasibility prior to large animal studies and clinical deployment. In the current study, we will present various surgical stages of bladder augmentation in both mice and rats using silk scaffolds and demonstrate techniques for awake and anesthetized cystometry.

\section{Video Link}

The video component of this article can be found at https://www.jove.com/video/3981/

\section{Protocol}

\section{Surgical Methods}

\section{Surgical Preparation and Anesthesia}

1. Set up the sterile surgical field with the necessary surgical instruments: shaving shears, forceps with teeth, fine atraumatic forceps, fine needle driver, gauze, Metzenbaum scissors, tenotomy scissors, scalpel blade, 30 gauge hypodermic needle, saline filled $1 \mathrm{~mL}$ syringe, four 6-0 polypropylene sutures, 7-0 polyglactin suture, 4-0 polyglactin suture. 
2. Anesthetize the animal with isoflurane inhalation in the induction chamber. Confirm complete induction of the animal before transferring to the surgical field. Ensure that the inhalational anesthesia tube is in appropriate position to have continuous anesthesia.

3. Place the animal supine on the sterile drape.

[For cystometric analysis, see section below in tunneling the cystostomy catheter.]

4. Use the shaving shears to remove the fur from the lower abdomen.

5. Prep the abdomen with betadine and $70 \%$ ethanol.

6. Prior to incision, an analgesic such as buprenorphine $(0.05-0.1 \mathrm{mg} / \mathrm{kg})$ can be injected subcutaneously for perioperative pain control.

\section{Incision and Exposure of the Bladder}

1. Make a 1-2 cm (dependant on size of animal and whether rat or mouse) lower midline incision with the scalpel through the skin. Deepen the incision at the lower portion of the incision through the rectus muscle taking care not to injure the underlying bowel or bladder.

2. Using toothed forceps, elevate the rectus muscle and dissect free the posterior surface of the muscle with fine Metzenbaum scissors.

3. Incise the remainder of the muscle in the midline for the entire length of your skin incision.

4. Deliver the bladder through the incisional wound (Figure 1). The bladder is usually the most dependent organ in the pelvis. (In the male, the prostate is actually more dependent and is larger than the decompressed bladder.)

5. Place one stay suture through the posterior wall of the bladder, and then another through the anterior wall of the bladder using 6-0 polypropylene suture. Place additional sutures laterally. Do not tie these sutures. When the sutures are held taut, the bladder will have a square configuration measuring approximately $1 \mathrm{~cm}^{2}$ (Figure 2). Be careful not to have too much tension on these sutures as they can easily be pulled through the bladder tissue.

6. Incise the bladder longitudinally through the anterior bladder wall (just inferior to the dome of the bladder) in the midline for approximately 1 $\mathrm{cm}(1.5-2 \mathrm{~cm}$ in the rat bladder).

\section{Anastomosis of the Scaffold}

1. Using fine scissors, trim the silk scaffold to the approximate area of the bladder defect.

2. Using 7-0 polyglactin suture, start at one corner of the scaffold and suture it to the bladder in a continuous, running fashion to create a watertight seal all the way around the defect (Figure 3).

3. Test the integrity of the anastomosis by filling the bladder with sterile saline by instilling it through the wall of the bladder with a 30 gauge hypodermic needle. If a leak is found, this can be closed with an additional interrupted 7-0 polyglactin suture to close the gap.

4. Reduce the reconstructed bladder back into the abdomen.

\section{Incisional Closure}

1. Prior to closure of the abdominal wall, inject the rectus muscle and subcutaneous tissue with bupivicaine for local anesthesia $(<3 \mathrm{mg} / \mathrm{kg}$ of $0.25 \%)$.

2. Reapproximate the rectus muscle with a continuous, running 4-0 polyglactin suture.

3. Close the skin with a continuous, running 4-0 polyglactin suture.

4. Clean and dry the incision (Figure 4).

5. Transfer the animal into a warm, clean cage for awakening from the anesthesia.

The steps for cystostomy catheter placement for cystometric analysis are as follows:

\section{Tunneling the Cystostomy Catheter}

1. Set up the sterile surgical field with the necessary surgical instruments: shaving shears, forceps with teeth, fine atraumatic forceps, fine needle driver, gauze, Metzenbaum scissors, tenotomy scissors, small curved clamp, scalpel blade, 6-0 polypropylene, 4-0 polyglactin suture, 3-0 silk suture (4-0 silk suture for mice), $18 \mathrm{G}$ needle, $22 \mathrm{G}$ blunt tip needle, $25 \mathrm{G}$ needle, $1 \mathrm{~mL}$ saline filled syringe, polyethylene tubing 50 (PE-50) cut for a length of $\sim 10 \mathrm{~cm}$

2. Flare the end of the PE-50 tubing by gently exposing it to a flame. Be careful not to melt the end off or occlude the lumen (this can be verified by injecting saline through a $25 \mathrm{G}$ needle connected to the "non-flared" end and ensuring flow). This serves as an anchor to maintain the tube within the bladder (Figure 5).

3. Anesthetize the animal as above in step 1.2.

4. Use the shaving shears to remove the fur from both the dorsum of the animal between the scapula and the lower abdomen on the ventrum.

5. Prep the areas with betadine and $70 \%$ ethanol. Place the animal prone on the drape.

6. Make a $1 \mathrm{~cm}$ incision on the dorsum between the scapula. Using the Metzenbaum scissors, develop a plane between the skin and the underlying muscle by placing the tips of the scissors in the plane and spreading them to create a tunnel around to the ventral abdomen.

7. Reposition the animal supine. Make your abdominal incision and expose the bladder as above in steps 2.1-2.4. Reduce the bladder back into the abdomen.

8. Place a small clamp into your subcutaneous tunnel created in step 5.6 starting from your dorsal skin incision. Using your fingers to protect the intra-abdominal contents, pierce through the abdominal wall with the tips of the clamp into the abdomen.

9. Grasp the smooth end of the PE-50 tubing with the clamp and pull it back through the dorsal incision. Ensure that the bulbed end is not pulled past the abdominal wall (Figure 6). 


\section{Placing the Cystostomy Tube}

1. Deliver the bladder through the incision. From this point on, the bladder should be handled with fine forceps to prevent trauma to the bladder that could cause damage or inflammation that can skew your cystometric results or result in additional discomfort for the animal postoperatively.

2. Make note of the proposed site for the cystostomy tube. It should be placed at the dome of the bladder (superior to the augment segment). This will prevent kinking or occlusion of the tube.

3. Using the 6-0 polypropylene suture, place a pursestring stitch at the dome of the bladder in the following manner: place the first throw through the wall of the bladder longitudinally, lateral to the proposed site for the cystostomy tube. Leave a small clamp on the loose end so that the suture is not inadvertently pulled all the way through. Place the next throw in a transverse direction, starting first going into the bladder wall slightly lateral to the exit of your first throw.

4. Do not pull the suture taut. Place your next suture parallel to your first (longitudinally) going into the bladder just cephalad to the exit site of your last, transverse suture. The fourth throw will start lateral to the exit of the last stitch and end next to the entrance for your very first throw. Done correctly, this forms a circumferential square around the proposed catheter site (Figure 7).

5. Using an $18 \mathrm{G}$ needle, pierce the bladder wall in the center of the pursestring suture. Be careful not to pierce too deeply (just enough to be intraluminal). Place the tips of your fine forceps in the opening and gently spread to widen the hole.

6. Insert the bulbed end of the catheter into the defect in the bladder until it is intraluminal. Pull the pursestring suture tight around the catheter and tie it down. This should cinch the bladder wall around the catheter keeping it in place (Figure 8).

7. Take one end of the suture and wrap it around the catheter once and tie this down to further secure the catheter.

\section{Testing the Catheter and Closing the Abdominal Incision}

1. With a $1 \mathrm{~mL}$ syringe and $25 \mathrm{G}$ needle, insert the needle into the tubing and slowly inject saline to distend the bladder. Observe for leaking around the catheter. Once you see leaking from the urethra, aspirate the saline to decompress the bladder again.

2. Close the abdominal incision as above in steps 4.1-4.4

\section{Closing the Dorsal Incision and Securing the Catheter (For rats)}

1. Reposition the animal prone.

2. Cut the catheter tubing at the level of the skin with the scissors. Insert a $22 G$ blunt tip needle into the tubing.

3. Close the skin over the tubing with 4-0 polyglactin in a running fashion. Leave the needle hub extruding from the skin.

4. Place an intravenous line cap on the blunt needle. Using 3-0 silk suture, secure the catheter tip to the skin (Figure 9).

5. Clean the incision. Transfer the rat into a warm, clean cage for awakening from the anesthesia.

\section{8.* Closing the Dorsal Incision and Securing the Catheter (For mice or rats)}

1. ${ }^{*}$ Occlude the distal end of the catheter by kinking it or using a flame to melt the end.

2. ${ }^{*}$ Coil the end of the tubing and leave it in the subcutaneous pouch on the dorsum of the animal (do NOT cut tubing to shorten it) (Figure 10).

3. ${ }^{*}$ Close the skin over the tubing with 4-0 polyglactin in a running fashion (Figure 11).

4. * On the day of cystometry, prepare the dorsal incision with betadine and $70 \%$ ethanol. Open the dorsal incision under anesthesia and remove the coiled tubing from the subcutaneous pouch. Close the incision. Awaken the animal from anesthesia and perform cystometry when it is fully awake.

\section{Representative Results - Surgical Methods}

The reconstructed bladder should be as water-tight as possible to avoid complications related to a significant urinary leak (Figure 3). Pain or discomfort usually manifest as shivering or scratching and gnawing at the abdominal incision. This can be managed with daily subcutaneous injections of a non-steroidal anti-inflammatory such as meloxicam (0.5-1.0 mg/kg subcutaneous). Typically, the animals only require the injections for the first 3 days post-operatively. This can be supplemented with an opioid, such as buprenorphine (0.05-0.1 mg/kg subcutaneous every 8-12 $\mathrm{hr}$ ) as needed. The animals should be monitored 3 times daily for the first 3 post-operatively days, twice daily for post-operative days $3-5$ and then daily thereafter to evaluate for pain, signs of infection, adequate wound healing, activity, grooming, and skin turgor. Antibiotics (Baytril, $5 \mathrm{mg} /$ $\mathrm{kg}$ subcutaneous every $24 \mathrm{hr}$ in a volume not exceeding $0.1 \mathrm{~mL}$ ) are given for the first $72 \mathrm{hr}$ following surgery, as surgical prophylaxis against infection. Signs of normal recovery are normal ambulation and activity levels, appropriate feeding and drinking, absence of pain or distress (no vocalization) and normal socialization with cagemates. A recovery time of at least 5-7 days should be given before cystometric analysis, to allow for bladder healing and decreased inflammation which could potentially affect the results.

Cystometric Analyses

\section{Awake Cystometric Analysis}

1. Setup described with MLT844 ADInstruments with data capture and analysis with LabChart v6 (ADInstruments) and infusion with a Harvard 22 syringe pump (Harvard Apparatus, Holliston, MA), although other comparable systems are available (Figure 12).

2. Calibrate both volume and pressure based on the specifications of the cystometric system used.

3. Place the animals in metabolic cages (cages with a wire mesh floor) which are suspended over a scale. The scale is connected to a transducer.

4. Purge the system of any air bubbles and ensure continuous flow from the infusion pump. 
5. Connect the data capture system to a computer and observe for data tracings. Adjust the scale accordingly. Bladder pressure and urine volume will be continuously recorded.

6. Access the suprapubic catheters with a $27 \mathrm{G}$ needle connected via a T-tube to the pressure transducer and the infusion pump. Begin the infusion of physiologic saline at $12.5 \mu \mathrm{L} / \mathrm{min}$ for the mouse and $100 \mu \mathrm{L} / \mathrm{min}$ for the rat.

7. Allow the voiding pattern tracing to stabilize (bladder pressure rise, followed by a void). This usually takes approximately $10-20$ minutes. Record the micturition cycles for 45-120 minutes or at least 3-4 voiding cycles.

8. Observe entire procedure in real-time to troubleshoot for complications that will lead to artifact (i.e. catheter kinking, obstruction, etc; see below discussion).

9. Stop the infusion, disconnect the catheter from the system, and return the animal to its cage.

\section{Unconscious Cystometric Analysis (No suprapubic catheter)}

1. Anesthetize the animal with urethane $(1-2 \mathrm{~g} / \mathrm{kg})$ intraperitoneal injection (IP)

2. Expose the bladder as above in steps 1.3-2.4.

3. Calibrate the system as in step 9.2. Prepare the system as in step 9.4-9.5.

4. Insert a $27 \mathrm{G}$ needle connected via a T-tube to the pressure transducer and infusion pump into the lateral aspect of the bladder.

5. Record the micturition cycles for $45-90$ minutes.

6. Stop the infusion, remove the needle from the bladder and euthanize the animal.

\section{Representative Results - Cystometric Analyses}

Urodynamic tracings can then be analyzed to derive parameters such as voided volumes, compliance, peak voiding pressures, inter contraction interval, micturition cycle time and post void residual volumes.

Cystometrogram can be divided into a filling and a voiding phase. A normal filling phase is the portion of the micturition cycle in which bladder fills with very little change in intravesical pressure. A normal voiding phase of the tracing consists of a steady rise in the intravesical pressure corresponding to the detrusor contraction. The highest pressure reached during the voiding phase of the tracing is termed the peak voiding pressure. A high peak voiding pressure could suggest an obstructive voiding pattern, a hypercontractile bladder or a kink in the SP catheter. Compliance can be calculated by acquiring the ratio of the volume instilled during the filling phase and the change in pressure (compliance $=\Delta \mathrm{V} /$ $\triangle \mathrm{P})$. A hypocompliant bladder is one that is unable to accommodate adequate urinary volumes at low pressures. The intercontraction interval can be calculated by analyzing the time between two contractions as seen on the cystometrogram. A short intercontraction interval is suggestive of an irritable bladder. The micturition cycle time refers to the time it takes for an entire filling and voiding phase to complete and can be easily ascertained by analyzing the tracing. At the conclusion of the cystometry, post-void residual (PVR) can be obtained. This is done by aspirating the suprapubic catheter upon the completion of a detrusor contraction. These parameters help the investigator objectively study the bladder dynamics as the bladder fills and empties.

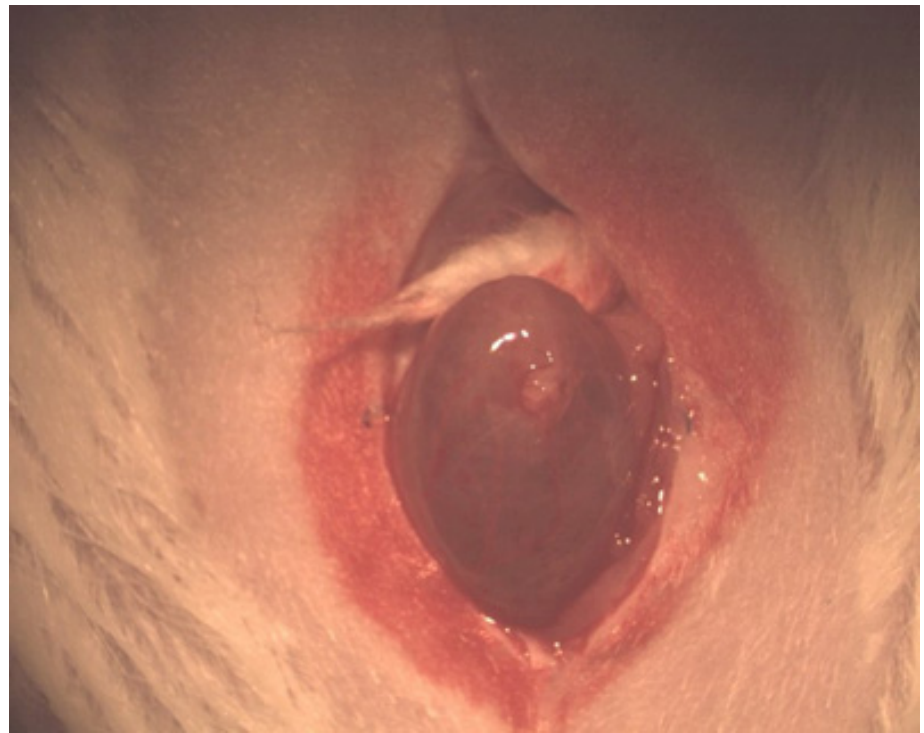

Figure 1. Photograph of the abdominal incision and extrusion of the bladder. 


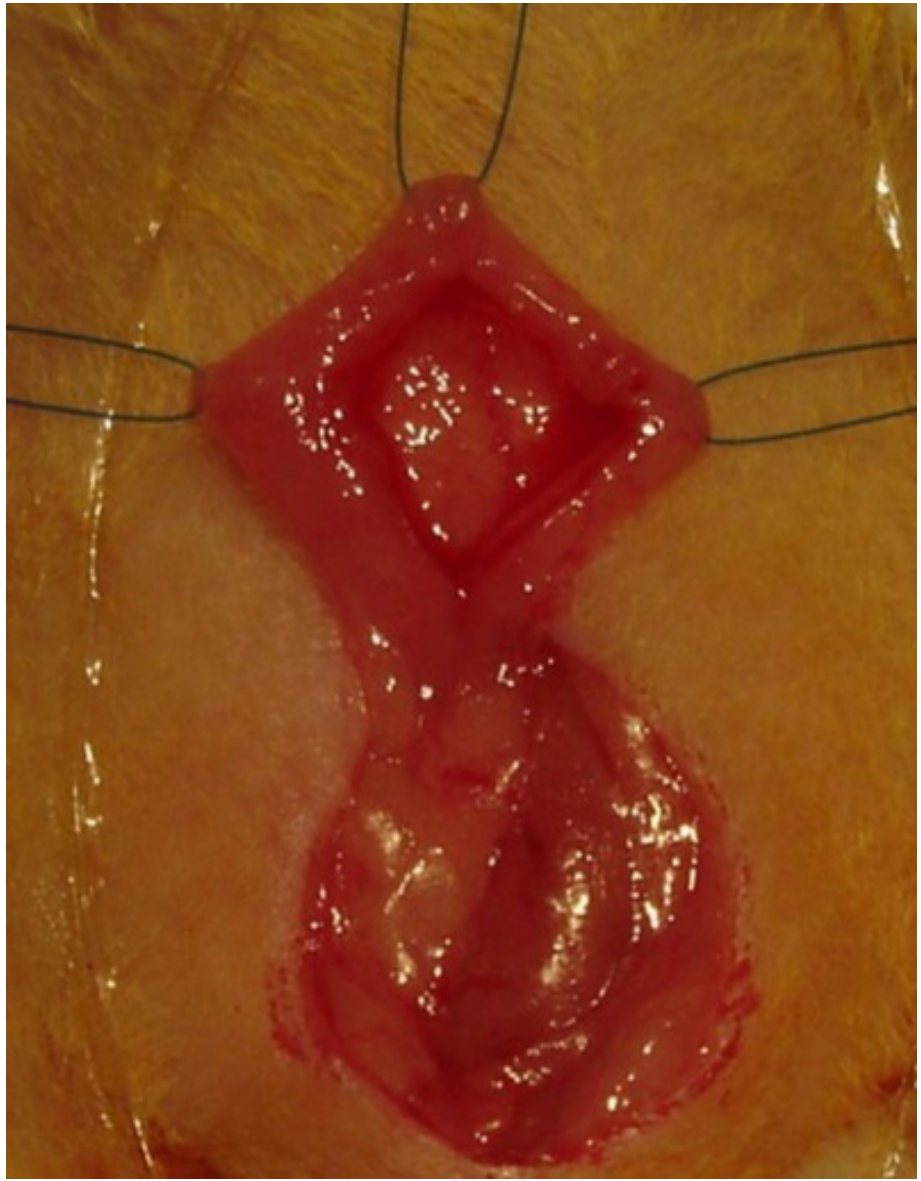

Figure 2. Bladder incision with exposure of the bladder lumen.

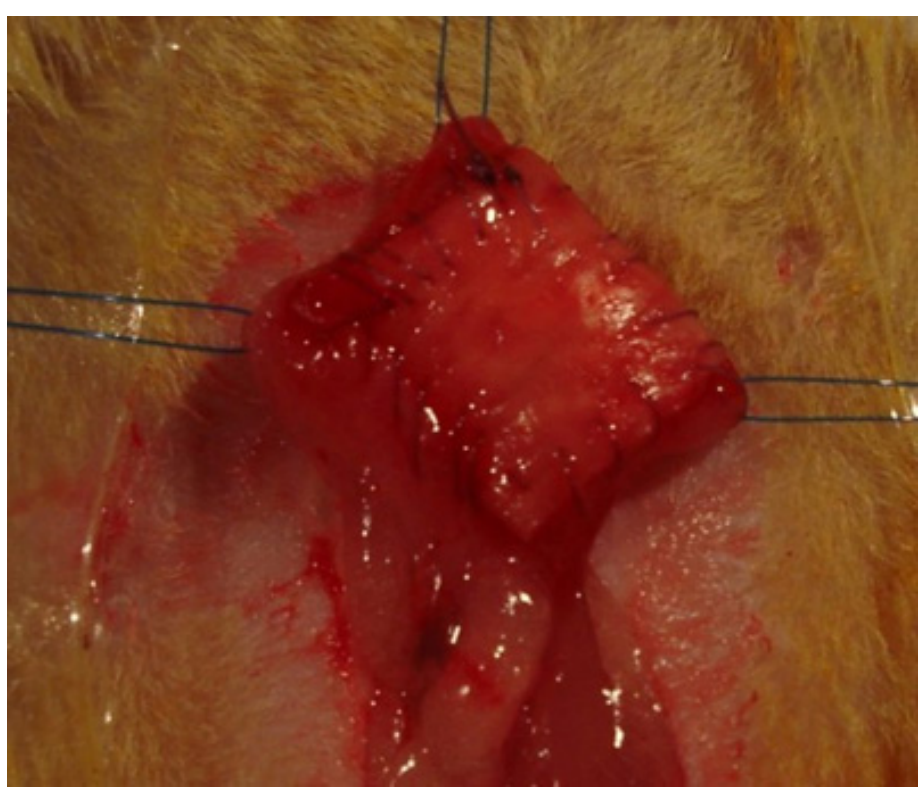

Figure 3. Integration of the implant onto the bladder wall. 


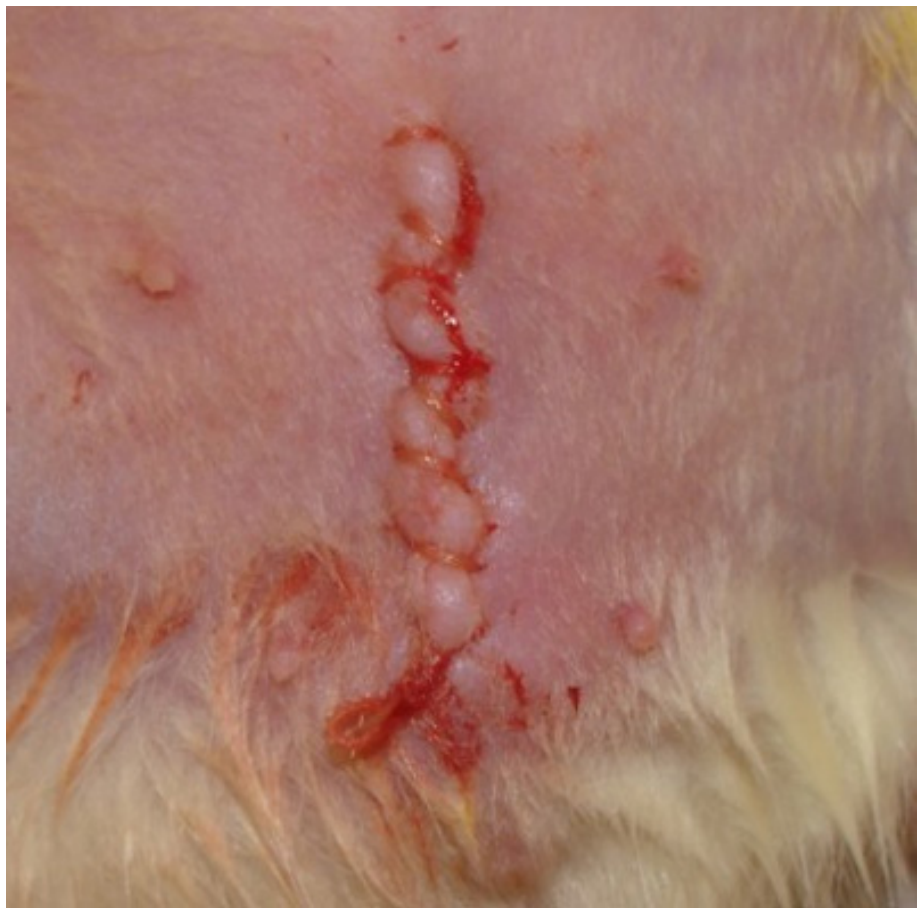

Figure 4. Photograph of the closed incision.

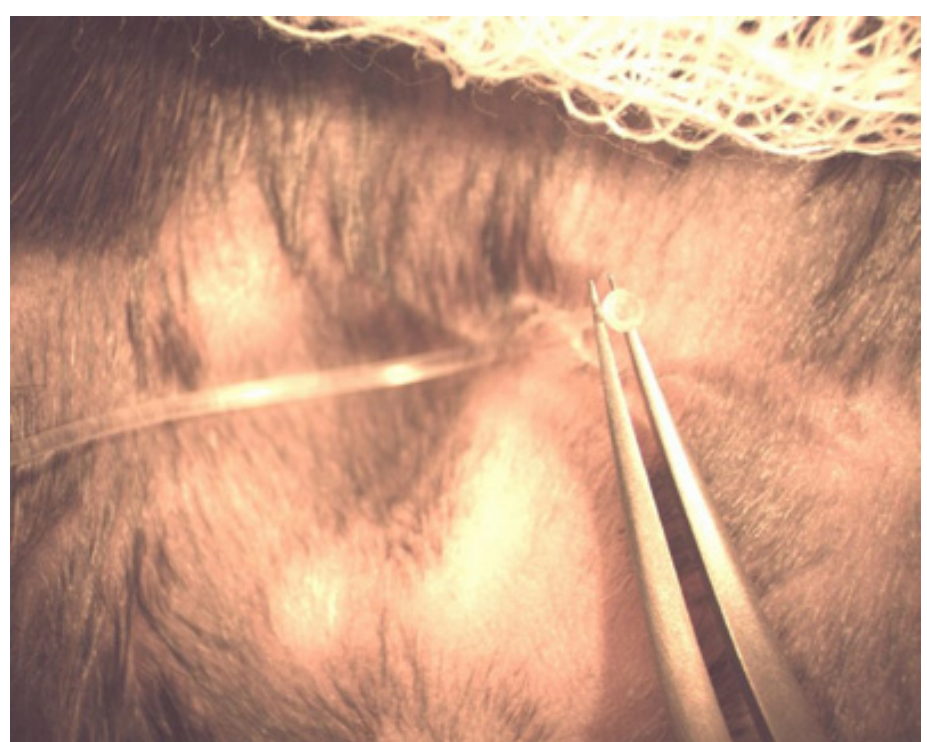

Figure 5. Flared end of the PE-50 tubing. 


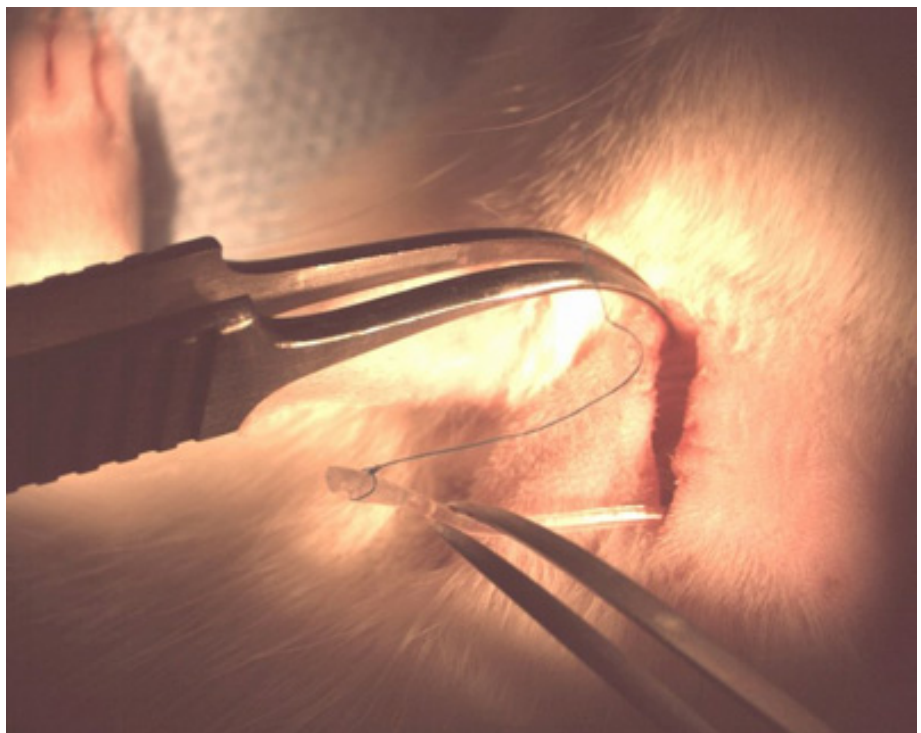

Figure 6. PE-50 tubing (catheter) through the dorsal incision.

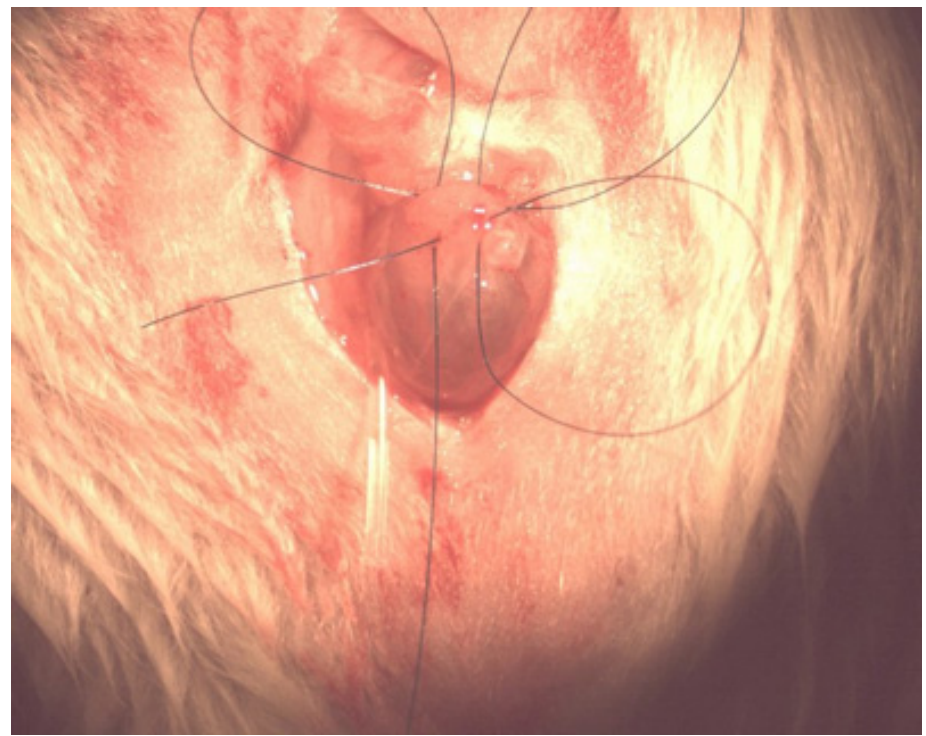

Figure 7. Pursestring suture.

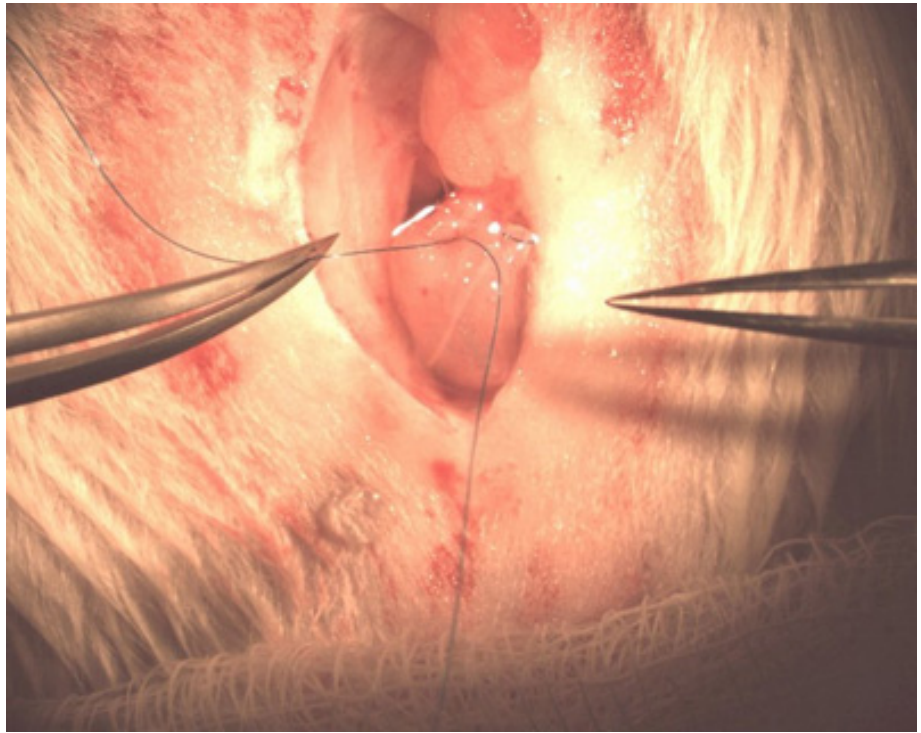


Figure 8. Securing the catheter to the bladder.

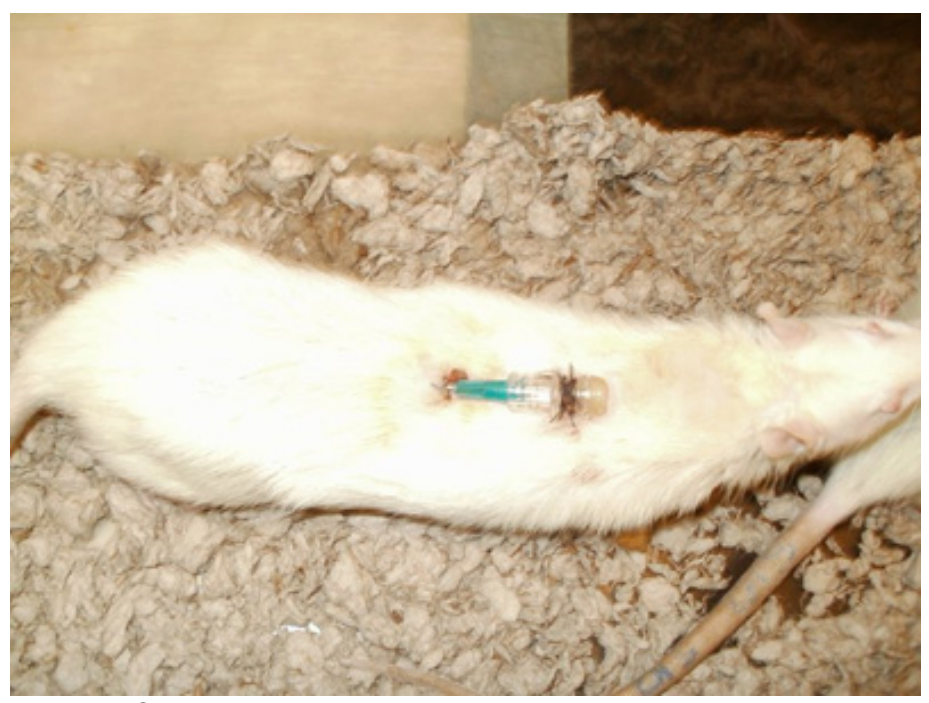

Figure 9. Secured catheter hub.

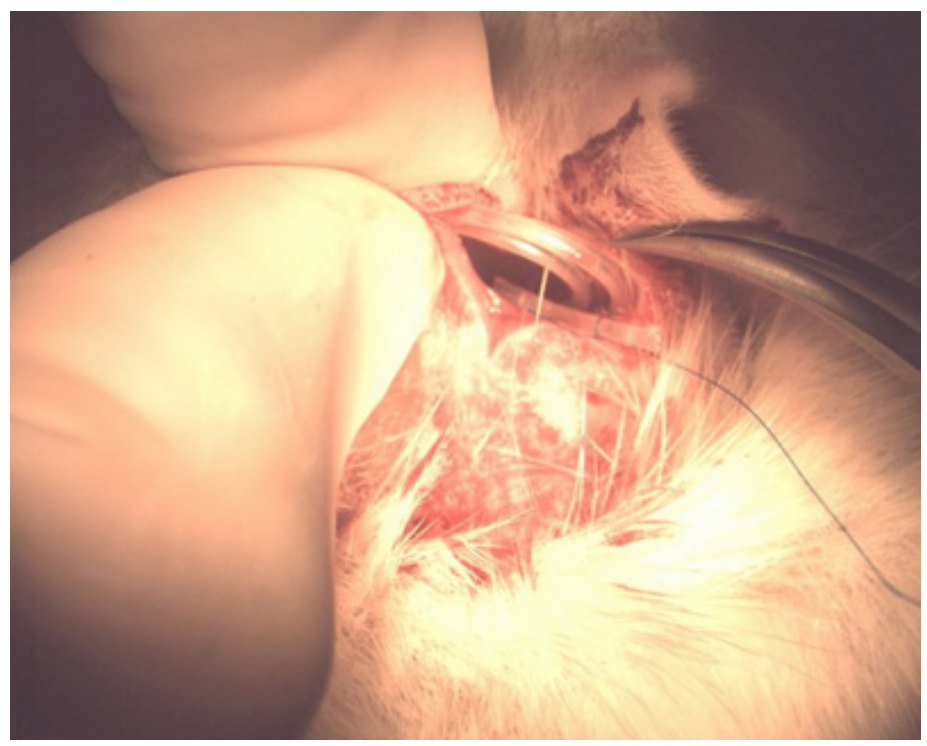

Figure 10. Coiled tubing in subcutaneous pouch. 


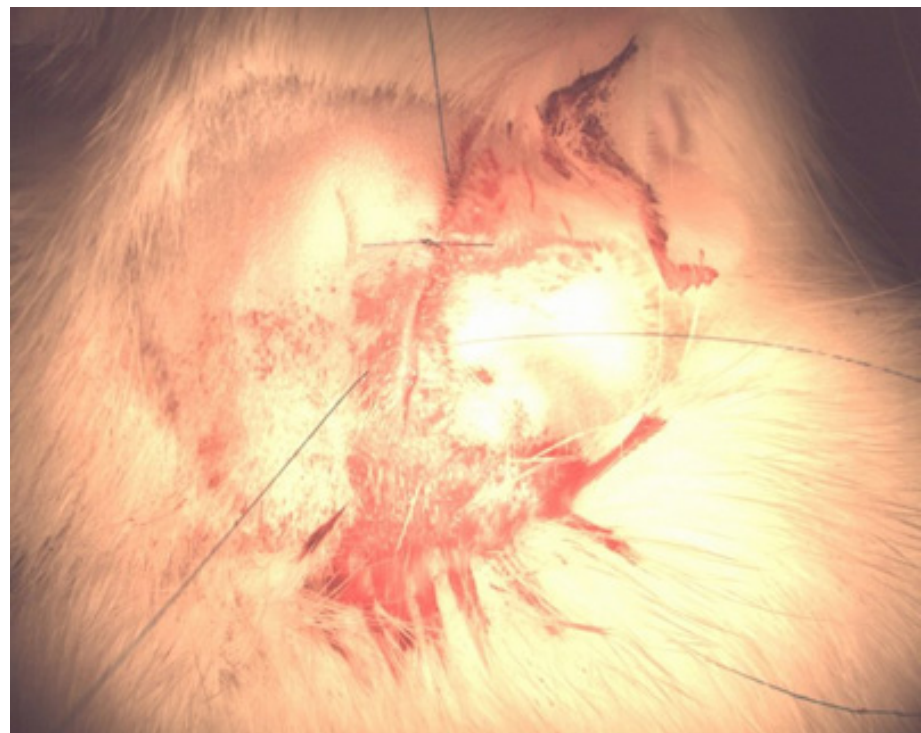

Figure 11. Dorsal incisional closure.

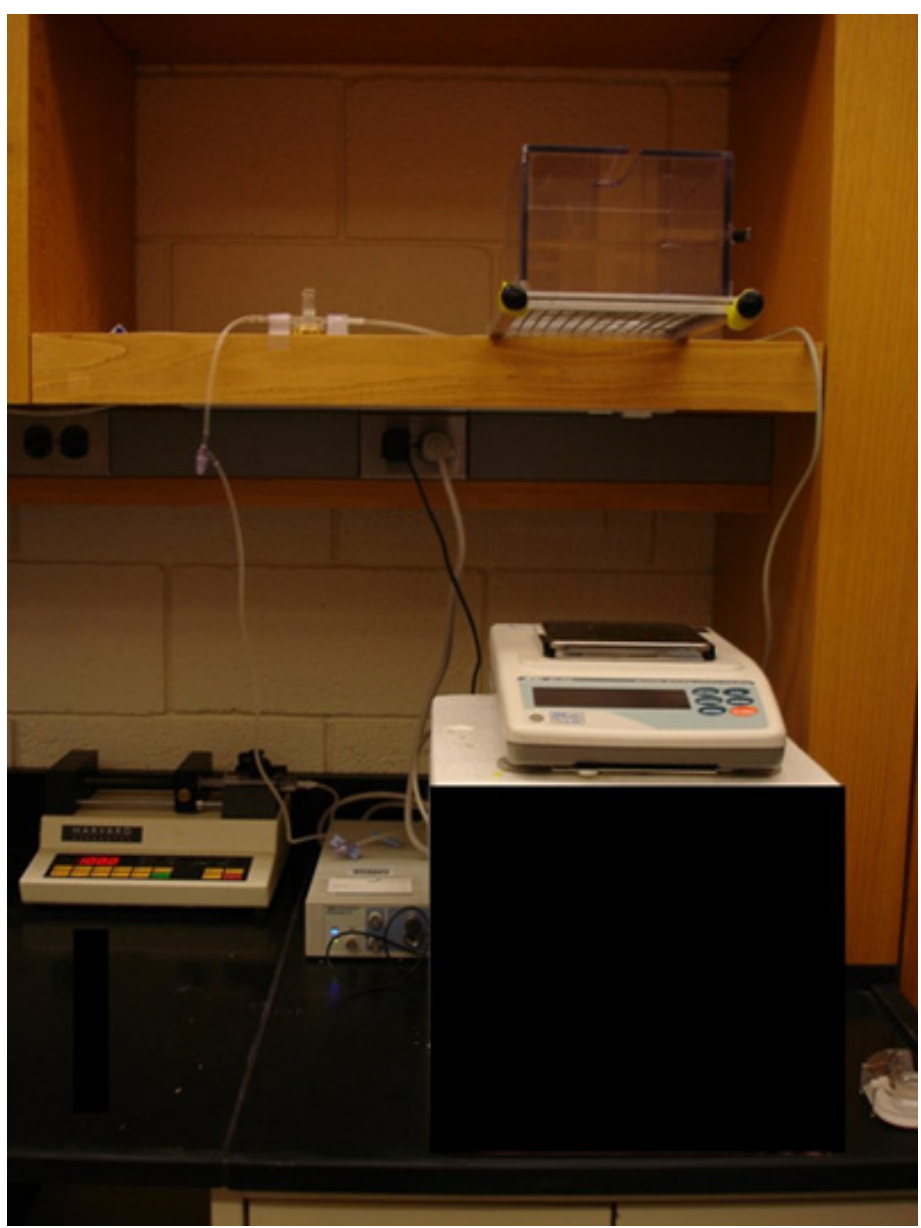

Figure 12. Example cystometric set-up. 


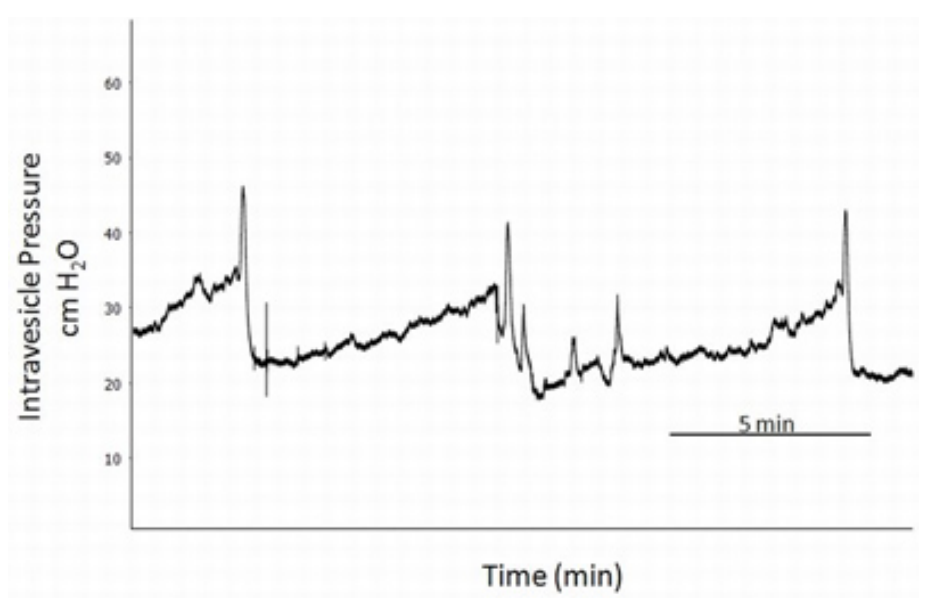

Figure 13. Representative cystometry tracing.

\section{Discussion}

Cystometric evaluations of biomaterial configurations following implantation and bladder augmentation in small animal models represents an important validation step in identifying optimal structural and mechanical characteristics of matrix designs for use in clinical situations. In this study, we describe surgical methods for performing bladder augmentation in mice and rats as well as cystometric techniques to determine urodynamic properties of engineered organs for functional assessments. We have utilized these techniques in multiple experiments involving both mice and rats, with each experiment consisting of $30+$ rodents without significant issues. Our research laboratory is a diverse conglomerate of basic scientists and physician surgeons, and surgeons with at least 5-6 years of post-graduate surgical training performed the procedural aspects of these experiments.

Regardless of the type of biomaterial used, the major difference between augmenting the bladder in rats versus mice is the size of the bladder. Due to smaller bladder size, dissection and incorporation of the biomaterial is more technically difficult in the mouse. To aid in visualization, a surgical microscope can be used. Since the size of the bladder in rats is larger, it is more amenable to situations where more than one procedure has to be performed on the bladder (e.g. augmentation and placement of cystostomy catheter). Additionally, the protocol above describes use of PE-50 tubing for the rat ${ }^{13}$, however, even larger size catheters, up to PE-100 have been used, especially for long-term studies ${ }^{14}$. In mice, a smaller caliber such as PE-10 tubing can be utilized ${ }^{15,16}$, but it should be kept in mind that smaller, more pliable tubes may not transmit pressure changes to the transducer accurately. Also, the alternative method of securing the catheter on the dorsum (step $8^{*}$ above) is done in mice due to their smaller body size and the blunt tip needle and IV cap are too cumbersome. The disadvantage of this is the need for anesthesia to extract the end of the catheter in the subcutaneous pouch prior to cystometry.

Studies have shown that in the initial first days (0-4 days) after placement of the catheters, cystometry revealed high bladder pressures and overactivity with low voiding volumes. These findings appeared to stabilize around the sixth to seventh day ${ }^{14,17}$ and therefore, is probably the ideal timing for cystometric evaluation. However, most reports in the literature perform cystometry within the first 3 days of catheterization ${ }^{18}$, and this accounts for the wide variation in the above parameters relative to time. Leaving the suprapubic catheter for a duration longer than 3 days carries with it morbidities such as risk of stones, dislodgement, infection, hematuria and occlusion of catheter with debris.

Different infusion rates during cystometry have been described from 1-3mL/hr for mice ${ }^{15,16}$ and $10-11 \mathrm{~mL} / \mathrm{hr}$ for rats ${ }^{13,19,20}$. Supraphysiologic infusion rates can cause falsely elevated pressures ${ }^{14}$. We use an infusion rate of $12.5 \mu \mathrm{L} / \mathrm{min}(0.75 \mathrm{~mL} / \mathrm{hr})$ for mice and $100 \mu \mathrm{L} / \mathrm{min}(6 \mathrm{~mL} /$ hr) for rats in our setup, but lower rates can also be utilized. The temperature of the physiologic saline should be at least room temperature, although warm $\left(37^{\circ}\right)$ saline is more optimal in order to avoid bladder overactivity provoked with instilling cold solution. In awake cystometry, it is crucial to allow for stabilization of the voiding pattern as the animal becomes adjusted to the cage, which in our experience requires a period of $\sim 10-20$ minutes. Following this, regular micturition cycles can be recorded for $45-120$ minutes or at minimum 3-4 voiding cycles. The animal should be observed in real-time since the animal is freely moving and complications such as twisting or kinking of the catheter can alter cystometric analysis. Limiting environmental noise during cystometry is desired to decrease animal movement and subsequent artifacts. Unconscious cystometry does not have the attendant problems as awake cystometry, but multiple anesthetics have been shown to inhibit spontaneous bladder contractions. This inhibition corresponds directly to the expected duration of action of the anesthetic drugs, i.e. when the anesthetic effect subsides, spontaneous contractions resume ${ }^{14}$. Moreover, pressures measured when the bladder overflowed, were statistically greater in anesthetized rats, both alive and post-mortem, indicating an effect on the passive compliance properties of the bladder wall. This effect is seen with pentobarbital ${ }^{21}$, ketamine, and chloralose IM/IP, in addition to inhaled halothane and intrathecal nesacaine ${ }^{14}$. A more extensive study of various anesthetics confirm this finding with suppression of the micturition reflex for both inhalational (isoflurane and methoxyflurane) and barbiturate (pentobarbital and thiobutabarbital) anesthetics under moderate anesthesia levels ${ }^{17}$. This effect was observed with even light or sedative levels of anesthesia with medications such as fentanyl-droperidol and ketamine-diazepam, and as in the previous study, as the anesthesia effect subsided, so did the inhibition ${ }^{17}$. For this procedure, urethane intraperitoneal injections can be used since it has been demonstrated that reflex micturition is preserved while also allowing for adequate anesthesia ${ }^{17,22}$. Moreover, no effect is observed with respect to micturition pressures ${ }^{23}$. Suprapubic catheter placement for cystometry is described here, since intraurethral catheterization has been shown to have higher bladder pressure curves and lower flow rates consistent with relative bladder outlet obstruction ${ }^{24}$. Moreover, intraurethral catheterization is only feasible in anesthetized animals, and even then, catheterization can be difficult, especially in male rodents and mice.

In conclusion, the choice of which model to use for bladder augmentation and/or cystometric analysis is dependent on the goals of the specific study. From a technical standpoint the rat model clearly holds the advantage for the reasons discussed above. However, the mouse model 
can be used in studies evaluating the roles of specific gene-encoded end products in diseases of the urinary tract, due to their susceptibility for genetic manipulation. This is not generally feasible in the rat.

Awake cystometry most accurately mimics the normal physiologic state in which these animals undergo their micturition cycles, and so, is likely to give a more reliable physiologic determination of bladder function. Moreover, the confounding variable of direct effects of anesthetics on bladder function is avoided.

\section{Disclosures}

No conflicts of interest declared.

\section{Acknowledgements}

These studies were funded, in part, by the Children's Hospital Boston Urology Endowment Revenue Fund and the National Institutes of Health grants NIBIB P41-EB002520 (Kaplan); NIDDK T32-DK60442 (Freeman); NIDDK 1K99-DK083616 (Mauney). We acknowledge Dr. Peter Zvara from the University of Vermont for assistance in establishing the technique for cystostomy tube placement and cystometry.

\section{References}

1. Atala, A. Tissue engineering for bladder substitution. World. J. Urol. 18, 364-370 (2000).

2. Roehrborn, C.G. Male lower urinary tract symptoms (LUTS) and benign prostatic hyperplasia (BPH). Med. Clin. North Am. 95, 87-100, [pii] S0025-7125(10)00148-3 doi:10.1016/j.mcna.2010.08.013 (2011).

3. Niknejad, K.G. \& Atala, A. Bladder augmentation techniques in women. Int. Urogynecol. J. Pelvic Floor Dysfunct. 11, 156-169 (2000).

4. Hensle, T.W. \& Gilbert, S.M. A review of metabolic consequences and long-term complications of enterocystoplasty in children. Curr. Urol. Rep. 8, 157-162 (2007).

5. Somani, B.K., et al. Bowel dysfunction after transposition of intestinal segments into the urinary tract: 8-year prospective cohort study. J. Urol. 177, 1793-1798, [pii] S0022-5347(07)00069-9 doi:10.1016/j.juro.2007.01.038 (2007).

6. Atala, A., Bauer, S.B., Soker, S., Yoo, J.J., \& Retik, A.B. Tissue-engineered autologous bladders for patients needing cystoplasty. Lancet. 367, 1241-1246, [pii] S0140-6736(06)68438-9 doi:10.1016/S0140-6736(06)68438-9 (2006).

7. Sharma, A.K., et al. A nonhuman primate model for urinary bladder regeneration using autologous sources of bone marrow-derived mesenchymal stem cells. Stem Cells. 29, 241-250, doi:10.1002/stem.568 (2011).

8. Chung, S.Y., et al. Bladder reconstitution with bone marrow derived stem cells seeded on small intestinal submucosa improves morphological and molecular composition. J. Urol. 174, 353-359, [pii] S0022-5347(05)60129-2 doi:10.1097/01.ju.0000161592.00434.c1 (2005).

9. Ashley, R.A., et al. Regional variations in small intestinal submucosa evoke differences in inflammation with subsequent impact on tissue regeneration in the rat bladder augmentation model. BJU Int. 105, 1462-1468, [pii] BJU8965 doi: 10.1111/j.1464-410X.2009.08965.x (2010)

10. Zhang, Y., Frimberger, D., Cheng, E.Y., Lin, H.K., \& Kropp, B.P. Challenges in a larger bladder replacement with cell-seeded and unseeded small intestinal submucosa grafts in a subtotal cystectomy model. BJU Int. 98, 1100-1105, [pii] BJU6447 doi: 10.1111/ j.1464-410X.2006.06447.x (2006).

11. Gomez, P., $3^{\text {rd }}$, et al. The effect of manipulation of silk scaffold fabrication parameters on matrix performance in a murine model of bladder augmentation. Biomaterials. 32, 7562-7570, [pii] S0142-9612(11)00747-2 doi:10.1016/j.biomaterials.2011.06.067 (2011).

12. Mauney, J.R., et al. Evaluation of gel spun silk-based biomaterials in a murine model of bladder augmentation. Biomaterials. 32, 808-818, [pii] S0142-9612(10)01248-2 doi: 10.1016/j.biomaterials.2010.09.051 (2011).

13. Persson, K., et al. Spinal and peripheral mechanisms contributing to hyperactive voiding in spontaneously hypertensive rats. Am. J. Physiol. 275, R1366-1373 (1998).

14. Yaksh, T.L., Durant, P.A., \& Brent, C.R. Micturition in rats: a chronic model for study of bladder function and effect of anesthetics. Am. J. Physiol. 251, R1177-1185 (1986).

15. Pandita, R.K., Fujiwara, M., Alm, P., \& Andersson, K.E. Cystometric evaluation of bladder function in non-anesthetized mice with and without bladder outlet obstruction. J. Urol. 164, 1385-1389, [pii] S0022-5347(05)67204-7 (2000).

16. Soler, R., Fullhase, C., Lu, B., Bishop, C.E., \& Andersson, K.E. Bladder dysfunction in a new mutant mouse model with increased superoxide--lack of nitric oxide? J. Urol. 183, 780-785, [pii] S0022-5347(09)02615-9 doi: 10.1016/j.juro.2009.09.074 (2010).

17. Matsuura, S. \& Downie, J.W. Effect of anesthetics on reflex micturition in the chronic cannula-implanted rat. Neurourol. Urodyn. 19, 87-99, doi:10.1002/(SICI)1520-6777(2000)19:1<87::AID-NAU9>3.0.CO;2-O (2000).

18. Andersson, K.E., Soler, R., \& Fullhase, C. Rodent models for urodynamic investigation. Neurourol. Urodyn. 30, 636-646, doi:10.1002/ nau.21108 (2011).

19. Soler, R., Fullhase, C., Santos, C., \& Andersson, K.E. Development of bladder dysfunction in a rat model of dopaminergic brain lesion. Neurourol Urodyn. 30, 188-193, doi:10.1002/nau.20917 (2011).

20. Streng, T., Santti, R., Andersson, K.E., \& Talo, A. The role of the rhabdosphincter in female rat voiding. BJU Int. 94, 138-142, doi:10.1111/ j.1464-4096.2004.04875.x BJU4875 (2004).

21. Malmgren, A., et al. Cystometrical evaluation of bladder instability in rats with infravesical outflow obstruction. J. Urol. 137, 1291-1294 (1987).

22. Smith, P.P. \& Kuchel, G.A. Continuous uroflow cystometry in the urethane-anesthetized mouse. Neurourol. Urodyn. 29, 1344-1349, doi:10.1002/nau.20850 (2010).

23. Cannon, T.W. \& Damaser, M.S. Effects of anesthesia on cystometry and leak point pressure of the female rat. Life Sci. 69, 1193-1202, [pii] S0024320501011821 (2001)

24. Smith, P.P., Hurtado, E., Smith, C.P., Boone, T.B., \& Somogyi, G.T. Comparison of cystometric methods in female rats. Neurourol. Urodyn. 27, 324-329, doi:10.1002/nau.20512 (2008). 